\title{
Management of burns
}

\author{
Priyanka Sehrawat ${ }^{1}$, Ishrat Yousuf ${ }^{1}$, Pranav Bansal ${ }^{2}$ \\ 1 - Resident; 2 - Professor\& Head, Department of Anesthesiology \& Critical Care, BPS Govt Medical College, \\ Khanpur Kalan, Sonepat, Haryana, India; E-mail: pranavbansal1@gmail.com
}

Anesthetists have dual responsibilities when encountered with burns patients; they are often called to share the initial management in Emergency Department in view of their expertise in IV access and airway management, in the ICU for further management including ventilation, and in $O R$ as patients with burns are regular visitors there.

Q1. Which of the following is false regarding carbon monoxide toxicity:-

(A) Carbon monoxide binds to hemoglobin 200 times more avidly than oxygen.

(B) There is rightward shift of oxy-hemoglobin dissociation curve

(C) Co-oximeter may measure carboxy-, meth-, deoxy-, and oxy-hemoglobin

(D) Pulse oximetry may show inaccurate results in burns patient

Q2. The initial stage of burn shock in burn patients results in all of the following except:-
(A) Increased capillary permeability
(B) Release of inflammatory mediators like TNF alpha and IL-1
(C) Decreased cardiac output
(D) Decreased systemic vascular resistance

Q3. Which of the following is false regarding cyanide poisoning:-

(A) Results in high anion gap metabolic acidosis

(B) May result in seizures and respiratory failure at levels $>100 \mathrm{ppm}$

(C) Cyanide stimulates intracellular cytochrome oxidase activity

(D) Intravenous sodium nitrate and sodium thiosulphate are useful in treatment

Q4. In pediatric burn patients which of the following formula is more appropriate in estimation of initial fluid requirement:
(A) Parkland formula
(B) Galveston formula
(C) Muir and Barclay formula
(D) Brooks formula

Q5. The intravenous fluid of choice for initial resuscitation of burn patients is:-
(A) $0.9 \%$ normal saline
(B) Ringers lactate
(C) Albumin
(D) Haemaccel (polygeline)

Q6. All of the following can cause early acute kidney injury in burn patients except:-
(A) Inadequate fluid resuscitation
(B) Poor renal perfusion
(C) Sepsis
(D) Myoglobinuria

Q7. Which of the following is not included in definition of major burns:-
(A) Second-degree burns involving $15 \%$ body surface area
(B) Chemical burns injury
(C) Electrical burns injury
(D) Inhalational burns injury

Q8. Which of the following statement is false regarding succinylcholine induced hyperkalemia in burn patients:-

(A) Potassium concentration starts to rise within the first minute of scoline administration, peaks within $5 \mathrm{~min}$, and starts to decline by $10-15 \mathrm{~min}$

(B) May lead to cardiac arrest

(C) Can be prevented by a prior defasciculation dose of NDMRs

(D) Scoline should be avoided after initial 24 hours till 2-years after burns injury 
Q9. Which of the following statement is correct with respect to drug dosing in a burns patient?

(A) Half the drug doses are required to achieve the desired effect

(B) Only the pharmacokinetics of drugs is affected by thermal injury

(C) Concentration of both plasma albumin as well alpha-1 acid glycoprotein decreases
(D) MAC values of volatile anesthetics may increase

Q10. Which of the following is not a component of 'burn pain paradigm' for pain management in burn patients?
(A) Background pain
(B) Breakthrough pain
(C) Chronic neuropathic pain
(D) Claudication pain

\section{EXPLANATIONS:}

A1 (B): Carbon monoxide binds to hemoglobin 200 times more avidly than oxygen forming carboxy-hemoglobin (COHb) binds to mitochondrial cytochrome oxidase and shifts the oxy-hemoglobin dissociation curve to left impairing oxygenation. Pulse oximetry is inaccurate in presence of $\mathrm{COHb}$ because it is interpreted as saturated hemoglobin. Cooximeter is required for the measurement of measure carboxy-, meth-, deoxy-, and oxy-hemoglobin.

A2 (D): The initial stage of burn shock in burn patients results in increased capillary permeability and leakage of protein rich plasma resulting in interstitial edema. The release of inflammatory mediators i.e. TNF alpha and IL-1 decreases cardiac output and increases systemic vascular resistance in early stage of burns.

A3 (C): Cyanide poisoning results in high anion gap metabolic acidosis. It manifests as headache, dizziness, tachycardia and tachypnea at levels $>50 \mathrm{ppm}$ may result in seizures and respiratory failure at levels $>100 \mathrm{ppm}$. Cyanide inhibits intracellular cytochrome oxidase activity. Management includes supportive treatment as oxygen administration, i.v. fluids, intravenous sodium nitrate and sodium thiosulphate.

A4 (B): Galveston formula is the most appropriate in calculating initial volume of fluid for resuscitation in pediatric burn patients. It uses $5 \%$ dextrose in ringers lactate $\left(5000 \mathrm{ml} / \mathrm{m}^{2}\right.$ of TBSA plus $2000 \mathrm{ml} / \mathrm{m}^{2}$ as maintenance fluid) to be administered in first $24 \mathrm{~h}$. One half is given in first $8 \mathrm{~h}$ and next half is given in next $16 \mathrm{~h}$. Dextrose is added to the resuscitation fluid in children so as to prevent hypoglycemia as children have very little glycogen stores.

A5 (B): Ringers lactate is the initial fluid of choice for resuscitation in burn injured patients.

A6 (C) Early AKI (occurring within first 5 days of burn injury) is due to inadequate fluid resuscitation, hypotension, inadequate renal perfusion, and myoglobinuria, while kidney injury in later stages is commonly due to sepsis and nephrotoxic drugs.

A7 (A): The criteria for classifying the extent of burn injury as major burn includes second-degree burns involving $25 \%$ body surface area in adults and $20 \%$ in children, third degree burns involving $10 \%$ body surface area, chemical burns injury, electrical burns injury, inhalational burns injury, burns involving eyes, ears, face, hands, feet, perineum and joints, burns associated with other major trauma (e.g. head injury, fractures) and patients with multiple co-morbidities.

A8 (C): The immature receptors of denervated burned tissue are more easily depolarized by scoline and produces hyperkalemia. The potassium concentration increases within first minute and peaks within 5 min and starts to decline by 10-15 min, that cannot be prevented by prior defasciculating dose of NDMRs. The rise in potassium levels does not correlate with the magnitude of burn injury and may lead to cardiac arrest.

A9 (D): MAC values of volatile anesthetics may increase and their duration of action may decrease. The key is to give titrated doses to have desired effect, both pharmacodynamics and pharmacokinetics of drugs are affected by thermal injury. In the early shock stage, the drug clearance and elimination reduces, whereas the same increases in hypermetabolichyperdynamic stage. Plasma albumin concentration decreases whereas concentration of alpha-1 gycoprotein, an acute phase reactant increases.

A10 (C): The five components of "Burn Pain Paradigm" include background pain, procedural pain, breakthrough pain, post-operative pain and chronic neuropathic pain. 